\title{
The Influence of Surgery Opportunity Choice on the Prognosis of Neonatal Necrotizing Enterocolitis
}

\author{
Jixue $\mathrm{Zhao}^{1}$, Xin $\mathrm{Fu}^{2, \text { a, * }}$ \\ ${ }^{1}$ Department of Pediatric Surgery, the First Hospital of Jilin University, Changchun, China \\ ${ }^{2}$ Nursing Administration Department, China-Japan Union Hospital of Jilin University, Changchun, \\ China \\ a jixuezhao0431@126.com
}

Keywords: NEC disease; surgery opportunity choice; Bell stage.

\begin{abstract}
Objective: To investigate the treatment effect of the different surgery opportunity choice on the NEC disease. Methods: A retrospective analysis of our Hospital in recent 8 years, neonatal and pediatric surgery in the diagnosis and treatment of neonatal necrotizing enter colitis cases. According to the modified Bell stage, Bell stage IIB 44 cases were divided into two groups: immediate operation group and non-immediate operation group. The cases of non-immediate operation group were treated conservatively, and would get surgical treatment when they get worse. The survival children were followed up to 6 months. Clinical data were retrospectively analyzed, including the survival rate and the incidence of incomplete intestinal obstruction Results: About 50\% of the Bell stage IIB cases developed into Bell stage III cases. The survival rate of the immediate operation group was obviously more than that of non-immediate operation group $(87.5 \% \mathrm{vs} 57.1 \%)(\mathrm{P}<0.05)$. And the incidence of incomplete intestinal obstruction of the immediate operation group was obviously less than that of non-immediate operation group $(14.3 \%$ vs56.3\%) $(\mathrm{P}<0.05)$. Conclusion: Early 1, NEC operation intervention can improve the survival rate of the NEC children obviously, and reduce the incidence of incomplete intestinal obstruction.
\end{abstract}

\section{Introduction}

Necrotizing enter colitis (NEC) of newborn is very common digestive system emergency. NEC developes rapidly, and has high fatality rate and more complications. The main pathological characteristics of NEC is the extensive hemorrhage necrosis of small intestine and the colon. NEC has higher morbidity and mortality rates among the premature and low birthweight. Morbidity and mortality rate of NEC is $1 \%-7.7 \%$ and $10 \%-30 \%$ in the US [1]. And the mortality rate of our country is $10 \%-50 \%$. Many NEC cases which have no intestinal necrosis and perforation can get conservative treatment during early stage, and many of them could get successful treatment which has the cure rate of $82.1 \%[2]$, but the data show that $20 \%-60 \%$ of the children still need the surgery[3].

At present, most of the doctors think that it is the best time for the operation when the intestinal wall full-thickness necrosis, but has not yet been perforated, that

Means Bell stage III cases, and the operation can excise the necrotic bowel, reduce

The toxin absorption, and relieve abdominal cavity infection. Because of the rapid progression and serious consequence of NEC, according to our experience, above-

Mentioned surgical indication always lead to more compications.

Maybe early1, NEC operation intervention can prevent disease progression and improve prognosis. Based on these considerations above, From August of 2010, we try to perform early operation (Bell stage IIB) for the NEC cases, and we have achived desirable effect. 


\section{Patient and Methods}

\subsection{Patient}

From January of 2010 to October of 2016, 44 infants with NEC of Bell stage IIB in the neonatology department, the First Hospital of Jilin University were enrolled in this retrospective analysis. Each patient signed an informed consent form. Approval was obtained from the institutional review committee of Jilin University.

\subsection{Study Design}

The patients were divided into two groups randomly: immediate operation group (experimental group) and non-immediate operation group (control group). The cases of non-immediate operation group were treated conservatively, and would get surgical treatment when they get worse. The survival children were followed up to 6 months.

We use the frequent operation methods, such as one-stage resection and anastomasis, enterostomy, enterectomy and enterostomy, abdominal cavity flushing and drainage. $[1,2,3,4]$

\subsection{Evaluation Criteria of Treatment Effects}

Evaluation criteria included the survival rate and the incidence of incomplete intestinal obstruction.

\subsection{Statistical Analysis}

All measured parameters including the survival rate and the incidence of incomplete intestinal obstruction, were analyzed by the statistical software program Statistical Product and Service Solutions (SPSS) 17.0 (SPSS Inc., Chicago, IL, USA) and expressed as mean \pm standard deviation $( \pm \mathrm{s})$, and t-test was used. Enumeration data including gender, prevalence frequency were analyzed by $\chi^{2}$ test. $\mathrm{P}<0.05$ was considered significant.

\section{Results}

\subsection{Baseline Characteristic}

There were no significant difference $(\mathrm{P}>0.05)$ in general data including operation age, gender between the two groups (Table 1.).

Table 1. Baseline characteristics of study patients

\begin{tabular}{cccc}
\hline Characteristic & control group $(16$ cases $)$ & experimental group $(28$ cases $)$ & $P$ value \\
\hline Operation age (days) & $9.1 \pm 2.6$ & $8.8 \pm 3.1$ & non-significant \\
Female & 6 & 10 & non-significant \\
Male & 10 & 18 & non-significant \\
\hline
\end{tabular}

\subsection{Evaluation of Treament Effects}

Among the cases of the immediate operation group (experimental group), 14 cases got full recovery, and the 2 remainders died, and the survival rate is $87.5 \%$; among the cases of the non-immediate operation group (experimental group), 16 cases got full recovery, and the 12 remainders died, and the survival rate is $57.1 \%$. The survival rate of experimental group was obviously higher than that of control group $(\mathrm{P}<0.05)$. The incidence of incomplete intestinal obstruction of the immediate operation group was obviously less than that of non-immediate operation group $(14.3 \%$ vs56.3 \%) $(\mathrm{P}<0.05)$, as is shown in Table 2. Postoperative follow-up was conducted for 6 months.

Table 2. Efficacy of the two groups

\begin{tabular}{cccc}
\hline Characteristic & control group(28 cases) & experimental group(16 cases) & $P$ value \\
\hline survival rate & $14 / 16$ & $16 / 28$ & $\mathrm{P}<0.05$ \\
The incidence of incomplete intestinal obstruction & $2 / 16$ & $9 / 28$ & $\mathrm{P}<0.05$ \\
\hline
\end{tabular}




\section{Discussion}

NEC is one of the most serious and common surgery urgent abdominal disease of the neonatal period, and it is more common in premature and low birthweight. NEC has many clinical manifestations, such as abdominal distension, vomiting, diarrhea, hematochezia. And at last, it always lead to multiple organs failure and even the shock. Case fatality rate is as high as $10 \%-50 \%$ [5-7]. Although many scholars had done many research about NEC, the case fatality rate did not decrease. Many NEC cases which have no intestinal necrosis and perforation can get conservative treatment during early stage, and many of them could get successful treatment. But the data show that most of the infants with NEC need surgery [3], and the early surgery is safe and effective [8].

It is important to choose the correct surgical indication and undergo the timely surgical interventions, which can avoid a series of complications and improving the prognosis [9]. In theory, it is the best time for the operation when the intestinal wall full-thickness necrosis, but has not yet been perforated, that means Bell stage III cases, and the operation can excise the necrotic bowel, reduce the toxin absorption, and relieve abdominal cavity infection. We always perform the conservative treatment for the NEC patients of Bell stage II B, and many of them has recovered. And about $50 \%$ of the Bell stage II B cases aggravated, including hypotension, bradyarrhythmia, frequent apnea, mixed acidosis, DIC, neutropenia, signs of peritonitis or Celiac free gas, and devoloped into Bell stage III, need operation. However many cases missed the optimal timing of surgery and lead to death. Because of the rapid progression and serious consequence of NEC, and the general deterioration, such as frequent apnea, bradyarrhythmia, infectious shock, multiple system organs failure, the survival cases always recovered slowly after operation and accompanied many complications. According to our experience, above-mentioned surgical indication tend to be late, maybe early operation intervention can prevent disease progression and improve prognosis. Based on these considerations above, we collected the stage II B cases for the early operation intervention. The survival rate of experimental group was obviously higher than that of control group $(87.5 \%$ vs $57.1 \%)$. The incidence of incomplete intestinal obstruction of the immediate operation group was obviously less than that of non-immediate operation group $(14.3 \% \mathrm{vs} 56.3 \%)$.

It is obviously that early operation intervention can improve the survival rate of NEC patients and reduce the postoperative complications. We analyze the possible reasons, on the one hand, the early stage cases always have the slight intestinal damage and less abdominal effusion, which will lead to the lower incidence rate of enterostenosis, intestinal adhesion and postoperative obstruction; on the other hand, the early stage cases always in good condition, and did not exist the electrolyte disturbance, infectious shock, so they can recover rapidly with less complications.

\section{Conclusion}

Early operation intervention can improve the survival rate of NEC patients and reduce the postoperative complications, So the early operation for the NEC cases is feasible in clinic.

\section{Acknowledgements}

Corresponding author: XIN FU E-mail: jixuezhao0431@126.com

\section{References}

[1]. Holman RC,Stoll BJ,Clarke MJ,et al.The epidemiology of necrotizing enterocolitis: infant mortality in the United States.Am J Pubic Health,1997,87(12):2026-2031

[2]. Chorna IS, Razumnyi PK, Buryi OM, et a1. Non-invasive methods of control of the intestinal motility in surgical practice [J]. Klin Khir. 1 999; (2): 22-25.

[3]. Thompson AM, Bizzarro MJ.Necrotizing enterocolitis in newborns: pathogenesis, prevention and management [J]. Drugs. 2008; 68(9):1227-38. 
[4]. Upadhyaya VD, Gangopadhyay AN, Pandey A, et al. Is pneumoperitoneum an absolute indication for surgery in necrotizing enterocolitis? [J] World J Pediatr. 2008 Feb; 4(1):41-4.

[5]. Thompson A, Bizzarro M, Yu S, et al. Risk factors for necrotizing enterocolitis totalis: a case-control study [J]. J Perinatol, 2011, 31 (11): 730-738.

[6]. Gephart SM, McGrath JM, Effken JA, et al. Necrotizing enterocolitis risk: state of the science [J]. Adv Neonatal Care, 2012, 12 (2): 77-87.

[7]. Diehl Jones ML, Askin DF, Nutritional modulation of neonatal outcome [J], AACN Clinical Issues, 2004, 15(1):83-96.

[8]. Gfroerer S, Fiegel H, Schloesser RL,et al. Primary laparotomy is effective and safe in the treatment of necrotizing enterocolitis[J]. World J Surg. 2014, 38(10):2730-2734.

[9]. Raval MV, Moss RL Current concepts in the surgical approach to necrotizing enterocolitisDl. Pathophysiology, 2014, 21(1): 105-110. 